\title{
Clinical and Nutritional Follow-up of Cats with Chronic Kidney Disease Fed with a Renal Prescription Diet
}

\author{
Gabriela da Cruz Schaefer, ${ }^{1,5}$, Silvana Bellini Vidor', Juliana Toloi Jeremias², \\ Cristiana Fonseca Ferreira Pontieri ${ }^{2}$, Marcio Antonio Brunetto ${ }^{3}$ \& Fernanda Vieira Amorim da Costa ${ }^{1,4}$
}

\begin{abstract}
Background: The use of prescription diets for cats with chronic kidney disease (CKD) is one of the main management approach of this disease in cats, and is considered a renoprotective strategy that may promote increased survival and/or improve quality of life, according to the stage of CKD. Besides that, nutritional assessment is important to monitor the maintenance of quality of life of the patients and their response to disease, especially those with chronic conditions. The aim of this study was to follow the clinical and nutritional status of cats with chronic kidney disease (CKD) IRIS stages II, III and IV fed with a renal prescription diet, followed for 12 months.

Materials, Methods \& Results: Patients were fed exclusively with a dry renal prescription diet and medications for the management of CKD were prescribed when needed. Exclusion criteria were cats that already received a renal prescription diet or medications for the treatment of CKD. Cats were evaluated every 2 months, considering body weight (BW), body condition score (BCS), muscle mass score (MMS), clinical and laboratory parameters. In all assessments, a complete blood count and biochemistry were performed by conventional methods with the patient fasted for $12 \mathrm{~h}$. In addition, urinalysis, urine protein:creatinine ratio (UPC) and urine culture were performed from a urine sample collected by cystocentesis. The quantitative variables were tested for their stability on consecutive assessments using the non-parametric Friedman test, and did not present significant variation during follow-up, except for systolic blood pressure (SBP). Eight cats with a diagnosis of CKD were included in the study and 6 of them remained in the same CKD stage during follow-up. On cat died due to an unrelated CKD cause. Regarding nutritional assessment, 5 of 7 cats maintained BW during the 12 months. Of these, 4 also maintained MMS and BCS. Three of 7 cats presented a decrease in MMS, 2 of which presented also a decreased BW and one maintained BW.

Discussion: IRIS staging results combined with Friedman's analysis demonstrated that the diet and the clinical management were effective in the non-progression of CKD in this study. As renal injury is not expected to be reversed in CKD, the maintenance of cats in the same IRIS stages and the minimum variation of the parameters is considered a positive result in this study. Hypertensive cats started on antihypertensive therapy during the study, achieving adequate control of SBP in most cases, what can justify the variation of this clinical parameter over the 12 months. Hyperphosphatemia was a frequent alteration, included stage II cats, and presented a positive response to nutritional and medical therapy. Despite CKD staging progression was not observed in most cats using serum creatinine as a single parameter, some cats presented BW and MMS reduction, which may have influenced this result. Weight loss and muscle wasting may have occurred by several reasons, including periods of hyporexia, presence of concomitant diseases, aging process or reduced protein content on renal prescription diets. This study enhances the importance of the association of clinical and nutritional management in the maintenance of cats with CKD. We suggest that other studies are done during longer periods of time and with a larger sample to support the results found. We also suggest new studies to evaluate the protein requirements for cats with CKD.
\end{abstract}

Keywords: body condition score, feline, muscle mass score, renal disease.

DOI: $10.22456 / 1679-9216.106220$ 


\section{INTRODUCTION}

Chronic kidney disease (CKD) is defined as the presence of structural or functional abnormalities of one or both kidneys that have been present for 3 months or longer and is considered one of the main causes of morbidity and mortality in cats [20]. It affects about $20 \%$ of cats and one third of cats with 15 years-old or more [14]. IRIS staging of CKD allows to manage factors that affect disease progression and provides information on its prognosis [8,9].

Several metabolic alterations are expected in patients with CKD, including azotemia, hyperphosphatemia, hypokalemia, metabolic acidosis, hypercalcemia or hypocalcemia [20]. Renal prescription diets recommend lower levels of phosphorus, moderate protein and sodium and higher levels of vitamin B, potassium, energy density, omega-3 and antioxidants, besides helps controlling metabolic acidosis [20]. There is a strong recommendation for the use of prescription diets in the management of cats with CKD IRIS stages II, III and IV, since nutritional therapy showed reduction of uremic episodes and mortality in randomized controlled clinical trials $[5,19,22]$. Thus, nutritional assessment is important to monitor the maintenance of quality of life of the patients and their response to disease, especially those with chronic conditions [6]. It is also important to measure the nutritional response to prescription diets using different methods, including body weight (BW), body condition score (BCS) and muscle mass score (MMS), associated with laboratory parameters [20].

The aim of this study was to describe clinical, nutritional and laboratory parameters of cats fed with a renal prescription diet, followed for 12 months.

\section{MATERIALS AND METHODS}

\section{Animals and exclusion criteria}

A prospective clinical study was conducted to evaluate cats with CKD IRIS stages II, III and IV fed exclusively with a dry formulation renal prescription $\operatorname{diet}^{1}$ [Premier Nutrição Clínica Renal Gatos (58.9 $\mathrm{g}$ protein/1000 kcal; $49.1 \mathrm{~g}$ fat/1000 kcal; $1.96 \mathrm{~g}$ EPA+DHA/1000 kcal; $1.1 \mathrm{~g}$ calcium $/ 1000 \mathrm{kcal}$ and 0.74 $\mathrm{g}$ phosphorus $/ 1000 \mathrm{kcal}$ )] and the amount of food offered to the animals was calculated with a prediction equation for the energy required for adult cat maintenance, as follows: $100 \times(\mathrm{BW})^{0.67}=\mathrm{kcal} /$ day [18]. Cats were admitted to the Veterinary Teaching Hospital (HCV) of the Universidade Federal do Rio Grande do Sul (UFRGS), located in Porto Alegre, Brazil, over a 12-month period. Exclusion criteria were cats that already received a renal prescription diet or medications for the treatment of CKD. Cats that did not accepted the diet or when owners were unable to feed them exclusively with it were also excluded. Owners were instructed to carry out gradual diet change over four weeks, by progressively increasing the proportion of the new diet.

\section{Assessments}

Cats were evaluated considering signalment, physical examination, nutritional status, laboratory and imaging tests before starting the study and every 2 months, for 12 months, except for imaging that was performed every 6 months. The initial IRIS stage of CKD was based on 2 values of serum creatinine concentration obtained at least 2 weeks apart, in a fasted and hydrated patient. After the initials physical and laboratorial exams, the prescription diet was introduced and, when necessary and according to each case, medications for the management of CKD were prescribed. In all assessments, a complete blood count $(\mathrm{CBC})$ and biochemistry were performed by conventional methods with the patient fasted for 12 h. In addition, urinalysis, urine protein:creatinine ratio (UPC):creatinine ratio (UPC) and urine culture were performed from a urine sample collected by cystocentesis. Proteinuria was considered when UPC $>0.4$ and borderline proteinuria when UPC $>0.2$ with absence of active urinary sediment and negative urine culture [8]. Serum phosphorus values were analyzed according to the stage of CKD (acceptable up to $4.5 \mathrm{mg} / \mathrm{dL}$ for stage II, up to $5.0 \mathrm{mg} / \mathrm{dL}$ for stage III and up to $6.0 \mathrm{mg} / \mathrm{dL}$ for stage IV) [9]. Systolic blood pressure (SBP) was measured using a Doppler device ${ }^{2}$, according to ACVIM guidelines [1]. Borderline hypertension was considered when SBP was between $150 \mathrm{mmHg}$ and $159 \mathrm{mmHg}$; hypertension when SBP was between 160 and $179 \mathrm{mmHg}$ and severely hypertension when SBP was above $180 \mathrm{mmHg}$ [8]. Abdominal ultrasonography and abdominal radiography (2 views) were performed to evaluate the urinary tract.

The BCS and MMS was assessed always by the same person. A 9-point BCS system [13] and a 4-point MMS system for cats [16] was used. Body weight (BW) variation after 12 months was calculated in relative values, according to the formula: [(final $\mathrm{BW}$-initial $\mathrm{BW}$ )/initial $\mathrm{BW}]^{*} 100$. Weight loss was 
considered when BW variation was above $10 \%$. Owners were told to measure the food intake of their cats and to stimulate their water intake.

\section{Clinical management}

Clinical management included fluid therapy to hydrate patients and to eliminate the pre-renal component of azotemia. This was done in the initial screening of patients, before the introduction of the prescription diet. Persistent hyperphosphatemia was treated with aluminum hydroxide $(30-60 \mathrm{mg} / \mathrm{kg} / \mathrm{day}$ PO, compounded formulation) administered with food. Amlodipine besylate ${ }^{3}$ was used for hypertension control (initial dose $0.625 \mathrm{mg} / \mathrm{cat} /$ day PO, adjusted as needed) and benazepril ${ }^{4}(0.25-0.5 \mathrm{mg} / \mathrm{kg} /$ day PO) for persistent renal proteinuria. Drugs for management of anorexia and vomiting included ranitidine $\mathrm{s}^{5}(1-2 \mathrm{mg} / \mathrm{kg}$ q12h PO), omeprazole ${ }^{6}(0.7 \mathrm{mg} / \mathrm{kg} /$ day PO), ondansetron $^{3}$ (0.5-1 mg/kg q12h PO), sucralfate ${ }^{7}$ (50-100 mg/ $\mathrm{kg} \mathrm{q} 12 \mathrm{~h}$ PO) and mirtazapine (1.88 mg/cat q48h PO, compounded formulation). Potassium citrate $(50 \mathrm{mg} /$ $\mathrm{kg}$ /day PO, compounded formulation) was prescribed in cases of hypokalemia.

\section{Statistical analysis}

Statistical analysis was performed using a statistical software ${ }^{8}$. The quantitative variables were tested for their stability, using the non-parametric Friedman test, to evaluate if the values on consecutive assessments were different. Significant results were considered when $P<0.05$. One patient was withdrawn from the statistical analysis by the occurrence of death during follow-up. A descriptive analysis of the initial and final values of the variables was performed.

\section{RESULTS}

Seven cats were excluded due to poor compliance in feeding the renal prescription diet exclusively during the study period or lack of acceptance of the new diet by the cat. Despite some owners said that there was no acceptance, it is not possible to ensure that the gradual transition to the new diet was carried out as recommended. The age of the 8 cats included in the study ranged from 4 to 15 years (mean $11 \pm 3.5$ ). Six of them were male and all of them were mixed breed and neutered. Drugs used in the clinical management of the 8 cats with CKD included in the study are described in Table 1.
Table 1. Drugs used in the clinical management of the eight cats with CKD included in the study.

\begin{tabular}{cc}
\hline Patient & Clinical management \\
\hline 1 & Omeprazole, ranitidine and potassium citrate \\
2 & Amlodipine and aluminum hydroxide \\
3 & Potassium citrate, amlodipine, aluminum hydroxide and \\
omeprazole
\end{tabular}

At the end of the 12-months, 1 cat progressed from stage II to III, and 1 died due to an unrelated CKD cause (pyothorax). Hence, 3 cats were classified as IRIS stage II, 3 cats as stage III, 1 cat as stage IV. Overall, 6 of 8 cats remained in the same stage (Table 2). Considering the substaging of proteinuria, except the cat on IRIS stage IV (P7) and the 1 who died at 8 months of follow-up (P8), all of the animals remained non-proteinuric or borderline proteinuric during the study (Table 2). All results of urine culture came up negative. Regarding arterial blood pressure, 5 of 8 cats were classified as hypertensive at the beginning. Of these 5 , only 1 remained hypertensive at the end of the study (Table 2).

Serum creatinine, phosphorus, urea, potassium and hematocrit values, UPC and USG values during the 12-month follow-up are shown in Figure 1 and did not present significant variation on consecutive assessments, by Friedman's test, during the study $(P>0.084)$.

Seven of the 8 cats $(87.5 \%)$ presented hyperphosphatemia at some point in the study. The highest values of serum phosphorus were observed in patients with IRIS stage IV (P7), however, Figure 1B shows that these values decreased progressively over time, after treatment with phosphate binders besides the diet.

Four cats $(50 \%)$ presented hypokalemia (potassium less than $3.5 \mathrm{mEq} / \mathrm{L}$ ) at some point in the study (Figure 1D). Only the IRIS stage IV patient (P7) maintained potassium concentrations persistently above the reference range during the study (mean $5.0 \mathrm{mEq} / \mathrm{L}$ ).

Of the 8 cats, $5(62.5 \%)$ presented isosthenuria in at least 2 evaluations (Figure 1G), including 3 cats IRIS stage III, 1 cat IRIS stage IV and 1 cat IRIS stage II. The patient with the highest urinary concentration was the youngest one (P1). 
Of the 8 cats, only P7 (12.5\%) presented mild anemia (hematocrit between 19-22\%), which persisted from before the follow-up until the eighth month of follow-up (Figure 1E).

Systolic blood pressure values (Figure 1H) presented significant variation over the 12 months by Friedman test analysis $(15.591 ; P=0.016)$. At a significance level of $5 \%$, there is evidence that the values are not all equal.

Regarding imaging tests, all cats presented alterations in the renal morphology by ultrasonographic examination. Two cats $(25 \%)$ presented renal cysts, $7(87.5 \%)$ showed signs compatible with chronic nephropathy, and $3(37.5 \%)$ presented nephrolithiasis. Three $(37.5 \%)$ cats presented renal pelvic dilatation at the ultrasound examination. The greatest measures of renal pelvic dilatation in these cats were: $0.42 \mathrm{~cm}, 0.39 \mathrm{~cm}$ and $0.28 \mathrm{~cm}$. Considering radiographic examination, nephrolithiasis was diagnosed in 2 cats $(25 \%)$, but none presented ureterolithiasis.

Regarding nutritional assessment, 5 of 7 (71.4\%) cats maintained BW during the 12 months. Of these, 4 also maintained MMS and BCS. Three of $7(42.8 \%)$ cats presented a decrease in MMS, 2 of which presented also a decreased BW and 1 maintained BW. According to the owners's perception, 5 of 7 cats $(71.4 \%)$ maintained their normal appetite after starting the prescription diet (Table 2). Figure 2 illustrates BW of each cat during 12 months of follow-up.

Table 2. Clinical, nutritional and laboratory parameters at baseline (T0) and end-point (12-months) of the study (T1) of the eight cats with CKD.

\begin{tabular}{|c|c|c|c|c|c|c|c|c|c|}
\hline Patient & & 1 & 2 & 3 & 4 & 5 & 6 & 7 & 8 \\
\hline \multirow{2}{*}{ IRIS staging } & T0 & II & II & III & III & II & II & IV & II \\
\hline & $\mathrm{T} 1$ & II & II & III & III & III & II & IV & Death \\
\hline \multirow{2}{*}{$\begin{array}{l}\text { IRIS substaging } \\
\text { (proteinuria) }\end{array}$} & T0 & NP & NP & NP & $\mathrm{BP}$ & NP & NP & $\mathrm{P}$ & $\mathrm{P}$ \\
\hline & $\mathrm{T} 1$ & NP & $\mathrm{NP}$ & $\mathrm{NP}$ & NP & $\mathrm{BP}$ & $\mathrm{NP}$ & $\mathrm{P}$ & Death \\
\hline \multirow{2}{*}{$\begin{array}{c}\text { IRIS substaging (blood } \\
\text { pressure) }\end{array}$} & T0 & $\mathrm{N}$ & $\mathrm{H}$ & $\mathrm{H}$ & $\mathrm{H}$ & $\mathrm{H}$ & $\mathrm{N}$ & $\mathrm{H}$ & $\mathrm{N}$ \\
\hline & $\mathrm{T} 1$ & $\mathrm{~N}$ & $\mathrm{~N}$ & $\mathrm{~N}$ & $\mathrm{H}$ & $\mathrm{N}$ & $\mathrm{N}$ & $\mathrm{N}$ & Death \\
\hline \multirow{2}{*}{$\begin{array}{l}\mathrm{BW} \\
(\mathrm{kg})\end{array}$} & T0 & 4.06 & 4.35 & 6.98 & 3.67 & 3.92 & 2.9 & 3.17 & 3.38 \\
\hline & $\mathrm{T} 1$ & 3.98 & 4.39 & $5.27^{\mathrm{a}}$ & 3.69 & 3.91 & 2.9 & $2.77^{\mathrm{a}}$ & Death \\
\hline \multirow{2}{*}{$\begin{array}{l}\text { MMS } \\
(0-3)\end{array}$} & T0 & 2 & 3 & 3 & 1 & 3 & 3 & 2 & 2 \\
\hline & $\mathrm{T} 1$ & 2 & 2 & 2 & 1 & 3 & 3 & 1 & Death \\
\hline \multirow{2}{*}{$\begin{array}{l}\mathrm{BCS} \\
(1-9)\end{array}$} & T0 & 5 & 6 & 8 & 4 & 5 & 4 & 3 & 5 \\
\hline & $\mathrm{T} 1$ & 5 & 5 & 8 & 4 & 5 & 4 & 4 & Death \\
\hline \multicolumn{2}{|l|}{ Appetite } & $\mathrm{M}$ & M & $\mathrm{D}$ & $\mathrm{M}$ & M & $\mathrm{M}$ & $\mathrm{D}$ & Death \\
\hline \multirow{2}{*}{ Systolic BP (mmHg) } & T0 & 150 & 160 & 184 & 160 & 170 & 126 & 166 & 144 \\
\hline & $\mathrm{T} 1$ & 140 & 150 & 140 & 160 & 130 & 120 & 140 & Death \\
\hline \multirow{2}{*}{ Creatinine (mg/dL) } & T0 & 2.7 & 1.8 & 3.5 & 3.1 & 2.2 & 2.4 & 5.5 & 2.1 \\
\hline & $\mathrm{T} 1$ & 1.9 & 1.7 & 4.6 & 3.7 & 3.0 & 2.8 & 5.0 & Death \\
\hline \multirow{2}{*}{$\begin{array}{c}\text { Phosphorus } \\
(\mathrm{mg} / \mathrm{dL})\end{array}$} & T0 & 2.7 & 3.7 & 5.4 & 5.7 & 4.5 & 3.8 & 12.2 & 5.1 \\
\hline & $\mathrm{T} 1$ & 3.4 & 5.4 & 4.0 & 5.3 & 4.1 & 4.6 & 5.8 & Death \\
\hline \multirow{2}{*}{ Ionized calcium $(\mathrm{mmol} / \mathrm{L})$} & T0 & 1.31 & 1.28 & 1.4 & 1.51 & 1.34 & 1.25 & 1.32 & 1.34 \\
\hline & $\mathrm{T} 1$ & 1.32 & 1.24 & 1.26 & 1.31 & 1.33 & 1.28 & 1.38 & Death \\
\hline \multirow{2}{*}{$\begin{array}{c}\text { Urea } \\
(\mathrm{mg} / \mathrm{dL})\end{array}$} & T0 & 49.4 & 49 & 81.8 & 99.4 & 44.4 & 59.4 & 216.6 & 44.2 \\
\hline & $\mathrm{T} 1$ & 52 & 55.3 & 97.3 & 110.4 & 71 & 63.9 & 122.4 & Death \\
\hline \multirow{2}{*}{ Potassium (mEq/L) } & T0 & 3.2 & 3.8 & 3.5 & 3.9 & 3.7 & 3.4 & 5.7 & 3.8 \\
\hline & $\mathrm{T} 1$ & 3.1 & 3.4 & 3.8 & 4.2 & 3.3 & 3.8 & 5.2 & Death \\
\hline \multirow{2}{*}{ Hematocrit (\%) } & T0 & 39 & 38 & 36 & 32 & 38 & 36 & 20 & 27 \\
\hline & $\mathrm{T} 1$ & 38 & 36 & 32 & 34 & 34 & 30 & 24 & Death \\
\hline \multirow{2}{*}{ UPC } & T0 & 0.008 & 0.068 & 0.085 & 0.23 & 0.037 & 0.094 & 5.57 & 2.4 \\
\hline & $\mathrm{T} 1$ & 0.011 & 0.022 & 0.12 & 0.103 & 0.36 & 0.17 & 1.14 & Death \\
\hline \multirow{2}{*}{ USG } & T0 & 1.064 & 1.032 & 1.020 & 1.014 & 1.038 & 1.036 & 1.014 & 1.010 \\
\hline & $\mathrm{T} 1$ & 1.080 & 1.040 & 1.012 & 1.016 & 1.012 & 1.014 & 1.010 & Death \\
\hline
\end{tabular}

$\mathrm{P}=$ proteinuric $\mathrm{BP}=$ borderline proteinuric; $\mathrm{NP}=$ non-proteinuric $\mathrm{N}=$ normotensive; $\mathrm{BH}=$ borderline hypertensive; $\mathrm{H}=$ hypertensive; $\mathrm{BW}=$ body weigh; $\mathrm{MMS}=$ muscle mass score; $\mathrm{BCS}=$ body condition score; $\mathrm{M}=$ maintenance; $\mathrm{D}=$ decrease. ${ }^{\mathrm{a}}$ Decrease in $\mathrm{BW}$ above $10 \%$. 
G.C. Schaefer, S.B. Vidor, J.T. Jeremias, et al. 2021. Clinical and Nutritional Follow-up of Cats with Chronic Kidney Disease Fed with a Renal Prescription Diet.

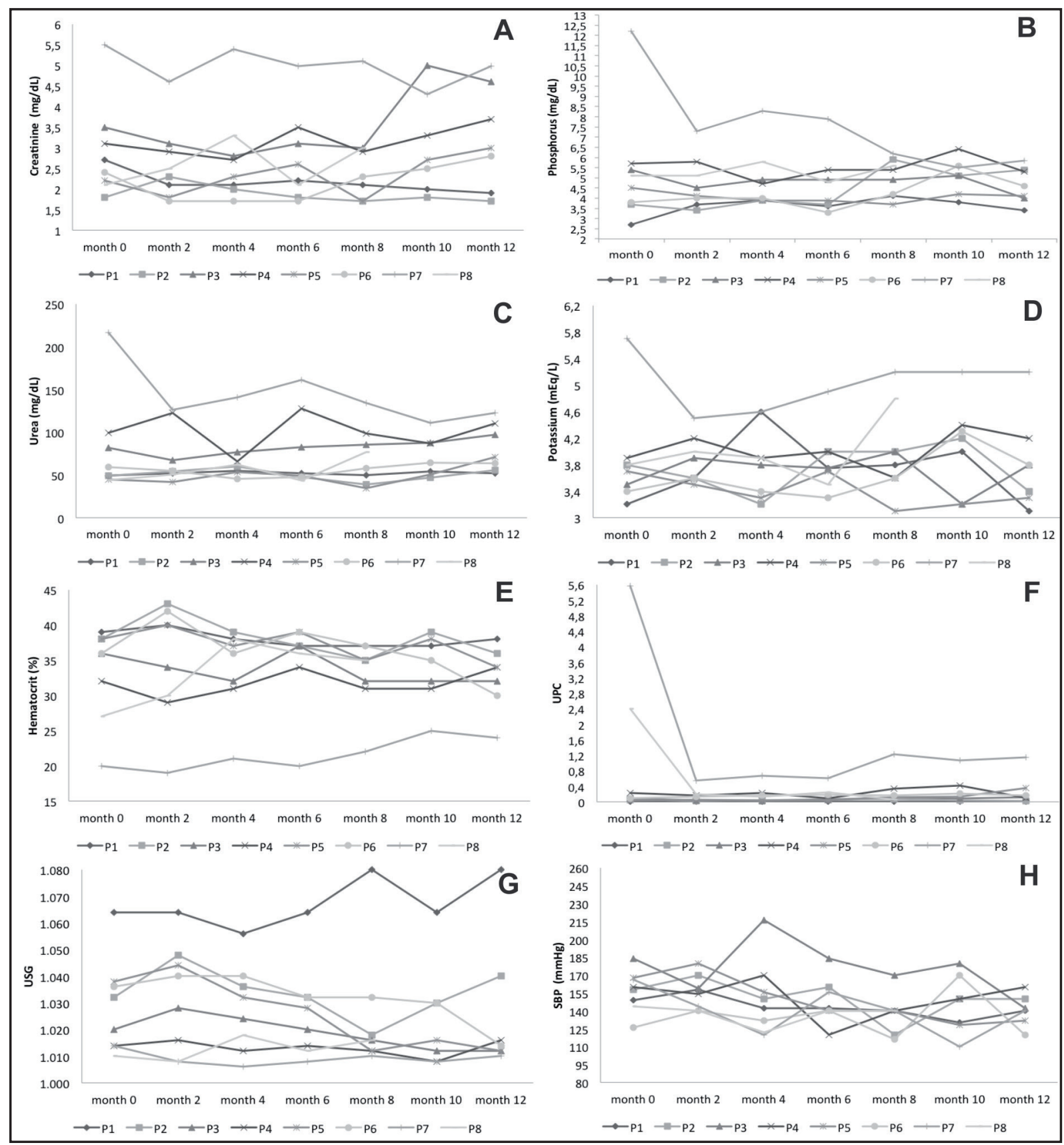

Figure 1. A- Serum creatinine; B- Phosphorus; C- Urea; D- Potassium; E- Hematocrit; F- UPC; G- USG and H- systolic blood pressure of the 8 cats with chronic kidney disease during the 12 months of follow-up.

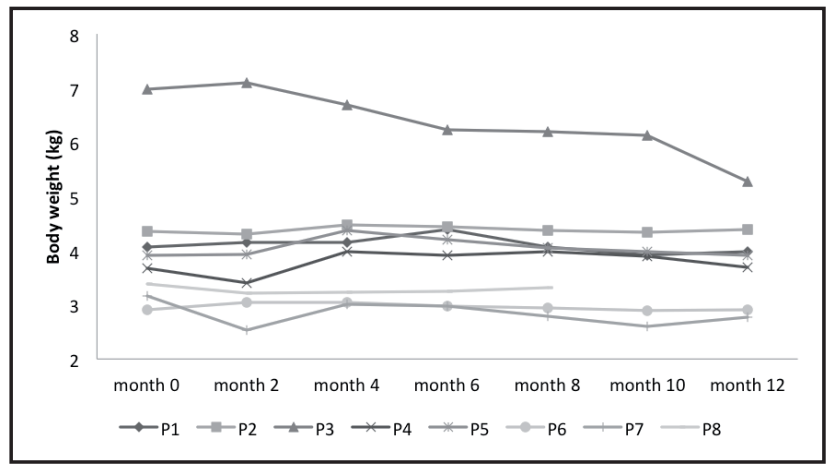

Figure 2. Body weight of the 8 cats with chronic kidney disease during the 12 months of follow-up. 


\section{DISCUSSION}

IRIS staging results combined with Friedman's analysis demonstrated that the diet and the clinical management were effective in the non-progression of $\mathrm{CKD}$, since these parameters did not change significantly throughout the study. The purpose of conservative management of CKD is to correct the abnormalities in hydroelectric, acid-base, endocrine and nutritional balance, in order to delay the progression of the disease $[20,23]$. Studies relate the use of a prescription diet with increased survival $[5,19,22]$ and prevention of the occurrence of uremia in renal patients [5,22]. Thus, renal injury is not expected to be reversed. Therefore, the maintenance of cats in the same IRIS stages and the minimum variation of the parameters is considered a positive result in this study. However, serum creatinine evaluation has limitations as a marker of kidney function, especially in patients with low MMS. On the other hand, symmetric dimethylarginine (SDMA) is not affected by muscle wasting, as serum creatinine is [7]. Two cats (P3 and P7) that remained at the same IRIS staging also presented a decrease in BW and MMS. This may have contributed to the maintenance of stable creatinine even with decrease of renal function, but it cannot be confirmed, since SDMA evaluation was not performed.

There are controversies regarding protein restriction in renal prescription diets for cats with CKD, as some authors question whether this restriction may contribute to the loss of muscle mass, which may lead to increased mortality [24]. Maintenance of MMS is important because the catabolism of muscle mass as an energy source increases the production of uremic toxins [2]. In the present study, 3 cats $(37.5 \%)$ had a 1-point reduction in MMS over 1-year period. Of these, 1 had a decrease in BCS and 2 in BW. This enhances the importance of doing nutritional assessment by more than one method, periodic weighing and individualized evaluation of each patient, in order to improve nutritional management before weight loss is significant. In addition, the nutritional state of patients with CKD can vary due to intercurrent diseases and periods of spontaneous reduction of food intake [17,21]. Anorexia represents the main clinical sign of cats with CKD [11]. Usually, these patients are also elderly, with simultaneous physiological reduction of digestibility of proteins and fats [21]. Thus, even small changes in protein metabolism can cause large losses of muscle mass [17]. Therefore, the loss of muscle mass in this study may be due to sev- eral factors other than protein content on the diet, such as aging process, concomitant diseases and decreased food intake. Maintaining the appetite of most cats and the adequate metabolizable energy content of the diet were determinant in keeping body weight with variation less than $10 \%$ in most of the cats. Only 2 cats (P3 and P7) were medicated with mirtazapine. Of the 2 cats that presented appetite reduction, one was the patient IRIS stage IV (P7) and the other was P3. The latter was the one that lost more weight, which can be justified by the reasons mentioned above.

Another important aspect of diet that may have influenced the results found is the high concentration of omega- 3 fatty acids. In the study published by Plantinga et al. [19], the authors found that the diet containing the highest EPA fatty acid concentration $(0.2-1.0 \mathrm{~g} / \mathrm{MJ})$ may have influenced the longer survival time of cats with CKD. Based on the recommendations of these authors, the EPA + DHA content present in the diet offered to the animals of the present study $(0.49 \mathrm{~g} / \mathrm{MJ})$ is similar to that observed in the study by Plantinga et al. [19].

Five of 8 cats were classified as hypertensive at the beginning of the study and only one remained hypertensive at the end of the study. A prevalence of $20 \%$ of hypertension in cats with CKD is reported [25]. Systemic arterial hypertension is considered a progression factor of kidney disease and is responsible for target organ damage, such as the kidneys, due to glomerular hypertension and glomerulosclerosis [23], demonstrating the importance of its diagnosis and treatment. Hypertensive cats started on antihypertensive therapy during the study, achieving adequate control of SBP, except for 1 case. The control of this clinical parameter and the decrease of its values can justify the independent course of this variable and its variation over the 12 months by Friedman test analysis. It was not possible to adequately control the SBP values of patient P3 during 6 months, even after institution of treatment with amlodipine besylate. This fact may justify the evolution of this patient IRIS staging and weight loss. However, because it is a clinical study, it was not possible to ensure that the owner has followed all medical recommendations, which could justify inadequate control of SBP. Furthermore, the patient should have received treatment with a second vasodilator, in an attempt to improve SBP control.

Considering the substaging of proteinuria, except the cat on IRIS stage IV (P7) and the 1 who died 
(P8), all of the animals remained non-proteinuric or borderline proteinuric (Figure 1F). The IRIS IV stage patient presented UPC above 5 in the first measurement but, in this occasion, the urine sediment was active, which may have contributed to this elevation. Urine culture of the same sample was negative. Glomerulonephritis was suspected and the cat was treated with benazepril, resulting in a decrease in UPC, but it still remained above the reference value [8]. The other patient with proteinuria, classified as IRIS stage II, had their values normalized after starting therapy with the same drug. Proteinuria of renal origin is a negative prognostic factor and is associated with interstitial fibrosis and tubular degeneration, and its treatment is indicated in all cases where UPC is above 0.4 [26].

Seven of the 8 cats $(87.5 \%)$ presented hyperphosphatemia at some point in the study. The highest values of serum phosphorus were observed in cats with IRIS stage IV (P7), however Figure 1B shows that these values decreased progressively over time, after treatment with phosphate binders and diet. Hyperphosphatemia is observed in about $60 \%$ of cats with $\mathrm{CKD}$ and its prevalence increases with a decrease in renal function [23]. In the early stages of the disease, high concentrations of parathyroid hormone tend to maintain normal phosphorus values. However, when the glomerular filtration rate decreases, this adaptive mechanism cannot be maintained, and hyperphosphatemia occurs. This alteration is common in IRIS stages III and IV of CKD and less common in stage II [10]. In this study, hyperphosphatemia was a frequent alteration, included stage II cats, and presented a positive response to nutritional and medical therapy.

Four cats (50\%) presented hypokalemia at some point in the study (Figure 1D). This alteration is related to polyuric CKD in cats [23]. Of these 4 cats, 2 presented isosthenuria, which corroborates the presence of concomitant polyuria. Only the IRIS stage IV patient (P7) maintained potassium concentrations persistently above the reference range during the study. Hyperkalemia may occur in patients with impaired renal function who are receiving an ACE inhibitor [12], which was $\mathrm{P} 7$ case.

Of the 8 cats, $5(62.5 \%)$ presented isosthenuria in at least 2 evaluations (Figure $1 \mathrm{G}$ ), including 3 cats IRIS stage III, 1 cat IRIS stage IV and 1 cat IRIS stage II. In a study with 46 cats, the mean USG decreased progressively with the evolution of the stages of CKD, being predominantly found in stages III and IV [15].
Of the 8 cats, only $\mathrm{P} 7$ presented mild anemia (hematocrit between 19-22\%), which persisted until the eighth month of follow-up (Figure 1E). Anemia in CKD is usually arregenerative, due mainly to the deficiency of erythropoietin [23]. The anemia of this patient was normocytic and normochromic, but since there was improvement without supplementation with the hormone, it was probably not related to CKD. For this reason, it is suspected that it was anemia of chronic inflammation [3], due to some concomitant condition, that has not been identified.

Imaging tests in cats with CKD are important both for diagnostic, especially in the early stages, and for the search of concomitant conditions, such as urolithiasis [20]. Renal pelvic dilatation may indicate pyelonephritis, renal disease, ureteral obstruction or may be seen in patients receiving fluid therapy [4]. Since no positive urine cultures were obtained and no ureterolithiasis were observed on radiographic and ultrasonographic examination, this alteration could be related only to excessive diuresis, due to polyuria [4].

The limitations of this study included those commonly found in clinical trials, such as owner failure related to comply with prescribed recommendations and lack of full control over the management of the patients. In addition, cats were followed for a relatively short time, since CKD in cats may remain stable over the years. Furthermore, renal biopsies were not performed, which made it difficult to determine the cause of CKD and its prognosis. The evaluation of renal function by SDMA would be more reliable for patients with loss of muscle mass. Still, the sample studied was small and the animals received medications for the management of CKD, so the effect of the prescription diet alone cannot be evaluated. However, it is believed that this is a study that represents the real conditions of clinical management of CKD in cats, including the challenges encountered by small animal clinicians.

\section{CONCLUSIONS}

The use of a renal prescription diet associated with the clinical management of CKD kept the cats clinically stable for 1 year long. Despite CKD staging progression was not observed in most cats using serum creatinine as a single parameter, some cats presented $\mathrm{BW}$ and MMS reduction. This study enhances the im- 
portance of the association of clinical and nutritional management in the maintenance of cats with CKD. We suggest that other studies are done during longer periods of time and with a larger sample to support the results found. We also suggest new studies to evaluate the protein requirements for cats with CKD.

\section{MANUFACTURERS}

${ }^{1}$ Premier Nutrição Clínica. Dourado, SP, Brazil.

${ }^{2}$ Med Mega. Franca, SP, Brazil.

${ }^{3}$ Biolab Sanus Farmacêutica Ltda. São Paulo, SP, Brazil.

${ }^{4}$ Elanco Saúde Animal. São Paulo, SP, Brazil.

${ }^{5}$ Aché Laboratórios Farmacêuticos S.A. Garulhos, SP, Brazil.

${ }^{6}$ União Química Farmacêutica Nacional S.A. São Paulo, SP, Brazil.

${ }^{7}$ EMS Sigma Pharma Ltda. Hortolândia, SP, Brazil.

${ }^{8}$ IBM Company. Columbus, OH, USA.
Funding. The authors are grateful for the financial support of the Premier Pet.

Acknowledgements. The authors gratefully acknowledge the contributions of: Comissão Comissão de Aperfeiçoamento de Pessoal do Nível Superior (CAPES), Ministério da Educação (MEC), Brazil and Hospital de Clínicas Veterinárias (HCV), Faculdade de Veterinária, Universidade Federal do Rio Grande do Sul (UFRGS).

Ethical approval. This study was approved by the Ethics Committee on the Use of Animals (CEUA) of the Universidade Federal do Rio Grande do Sul (UFRGS) under protocol number no. 25809.

Declaration of interest. Cristiana F.F. Pontieri and Juliana T. Jeremias are employees of Premier Pet. The other authors do not have any potential conflicts of interest to declare other than conducting this survey funded by Premier Pet.

\section{REFERENCES}

1 Acierno M.J., Brown S., Coleman A.E., Jepson R.E., Papich M., Stepien R.L. \& Syme H.M. 2018. ACVIM consensus statement: Guidelines for the identification, evaluation, and management of systemic hypertension in dogs and cats. Journal of Veterinary Internal Medicine. 32(6): 1803-1822. DOI: 10.1111/jvim.15331

2 Barber P. 2003. Diagnosis and management of chronic renal failure in the cat. In Practice. 25(6): 306-313. DOI: 10.1136/inpract.25.6.306

3 Chikazawa S. \& Dunning M.D. 2016. A review of anaemia of inflammatory disease in dogs and cats. Journal of Small Animal Practice. 57(7): 348-353. DOI: 10.1111/jsap.12498

4 D'Anjou M.-A., Bédard A. \& Dunn M.E. 2011. Clinical significance of renal pelvic dilatation on ultrasound in dogs and cats. Veterinary Radiology \& Ultrasound. 52(1): 88-94. DOI: 10.1111/j.1740-8261.2010.01729.x

5 Elliott J., Rawlings J.M., Markwell P.J. \& Barber P.J. 2000. Survival of cats with naturally occurring chronic renal failure: effect of dietary management. Journal of Small Animal Practice. 41(6): 235-242. DOI: 10.1111/j.17485827.2000.tb03932.x

6 Freeman L., Becvarova I., Cave N., MacKay C., Nguyen P., Rama B., Takashima G., Tiffin R., van Beukelen P. \& Yathiraj S. 2011. WSAVA Nutritional Assessment Guidelines. Journal of Feline Medicine and Surgery. 13(7): 516-525. DOI: 10.1016/j.jfms.2011.05.009

7 Hall J.A., Yerramilli M., Obare E., Yerramilli M., Yu S. \& Jewell D.E. 2014. Comparison of serum concentrations of symmetric dimethylarginine and creatinine as kidney function biomarkers in healthy geriatric cats fed reduced protein foods enriched with fish oil, L-carnitine, and medium-chain triglycerides. The Veterinary Journal. 202(3): 588-596. DOI: $10.1016 / j . t v j 1.2014 .10 .021$

8 IRIS. International Renal Interest Society. 2019. IRIS Staging of CKD. Available from: <http://www.iris-kidney. com/pdf/IRIS_Staging_of_CKD_modified_2019.pdf>. [Accessed online in July 2019].

9 IRIS. International Renal Interest Society. 2019. Treatment Recommendations for CKD in Cats. Online. Available from: <http://www.iris-kidney.com/pdf/IRIS_CAT_Treatment_Recommendations_2019.pdf>. [Accessed online in July 2019].

10 Kidder A. \& Chew D. 2009. Treatment options for hyperphosphatemia in feline CKD: what's out there? Journal of Feline Medicine and Surgery. 11(11): 913-924. DOI: 10.1016/j.jfms.2009.09.012

11 King J.N., Tasker S., Gunn-Moore D.A. \& Strehlau G. 2007. Prognostic factors in cats with chronic kidney disease. Journal of Veterinary Internal Medicine. 21(5): 906-916.

12 Kogika M.M. \& Morais H.A. 2008. Hyperkalemia: a quick reference. Veterinary Clinics of North America: Small Animal Practice. 38(3): 477-480. DOI: 10.1016/j.cvsm.2008.01.024 
13 Laflamme D.P. 1997. Development and validation of a body condition score system for cats: a clinical tool. Feline Practice. 25(5): 13-18.

14 Lund E.M., Armstrong P.J., Kirk C.A., Kolar L.M. \& Klausner J.S. 1999. Health status and population characteristics of dogs and cats examined at private veterinary practices in the United States. Journal of the American Veterinary Medical Association. 214(9): 1336-1341.

15 McLeland S.M., Cianciolo R.E., Duncan C.G. \& Quimby J.M. 2015. A comparison of biochemical and histopathologic staging in cats with chronic kidney disease. Veterinary Pathology. 52(3): 524-534. DOI: 10.1177/0300985814561095

16 Michel K.E., Anderson W., Cupp C. \& Laflamme D. 2009. Validation of a subjective muscle mass scoring system for cats. Journal of Animal Physiology and Animal Nutrition. 93(6): 802-810.

17 Mitch W.E. 2007. Malnutrition is an unusual cause of decreased muscle mass in chronic kidney disease. Journal of Renal Nutrition. 17(1): 66-69. DOI: 10.1053/j.jrn.2006.10.010

18 NRC. National Research Council. 2006. Nutrient Requirements of Dogs and Cats. Washington, DC: National Academies Press, 424p.

19 Plantinga E.A., Everts H., Kastelein A.M.C. \& Beynen A.C. 2005. Retrospective study of the survival of cats with acquired chronic renal insufficiency offered different commercial diets. The Veterinary Record. 157(7): 185-187. DOI: 10.1136/vr.157.7.185

20 Polzin D.J. 2011. Chronic kidney disease. In: Bartges J. \& Polzin D. (Eds). Nephrology and Urology of Small Animals. Ames: Wiley-Blackwell, pp.433-471.

21 Polzin D.J. \& Churchill J.A. 2016. Controversies in veterinary nephrology: renal diets are indicated for cats with International Renal Interest Society chronic kidney disease stages 2 to 4: the pro view. Veterinary Clinics of North America: Small Animal Practice. 46(6): 1049-1065. DOI: 10.1016/j.cvsm.2016.06.005

22 Ross S.J., Osborne C.A., Kirk C.A., Lowry S.R., Koehler L.A. \& Polzin D.J. 2006. Clinical evaluation of dietary modification for treatment of spontaneous chronic kidney disease in cats. Journal of the American Veterinary Medical Association. 229(6): 949-957. DOI: 10.2460/javma.229.6.949

23 Roudebusch P., Polzin D.J., Ross S.J., Towell T.L., Adams L.G. \& Forrester S.D. 2009. Therapies for feline chronic kidney disease: what is the evidence? Journal of Feline Medicine and Surgery. 11(3): 195-210. DOI: 10.1016/j. jfms.2009.01.004

24 Scherk M.A. \& Laflamme D.P. 2016. Controversies in veterinary nephrology: renal diets are indicated for cats with International Renal Interest Society chronic kidney disease stages 2 to 4: the con view. Veterinary Clinics of North America: Small Animal Practice. 46(6): 1067-1094. DOI: 10.1016/j.cvsm.2016.06.007

25 Syme H.M., Barber P.J., Markwell P.J. \& Elliott J. 2002. Prevalence of systolic hypertension in cats with chronic renal failure at initial evaluation. Journal of the American Veterinary Medical Association. 220(12): 1799-1804. DOI: 10.2460/javma.2002.220.1799

26 Vaden S.L. \& Elliott J. 2016. Management of proteinuria in dogs and cats with chronic kidney disease. Veterinary Clinics of North America: Small Animal Practice. 46(6): 1115-1130. DOI: 10.1016/j.cvsm.2016.06.009 